\title{
On generalized Eisenstein series and Ramanujan's formula for periodic zeta-functions
}

\author{
M. Cihat Dağlı and Mümün Can \\ Department of Mathematics, Akdeniz University, \\ 07058-Antalya, Turkey \\ e-mails: mcihatdagli@akdeniz.edu.tr, mcan@akdeniz.edu.tr
}

\begin{abstract}
In this paper, transformation formulas for a large class of Eisenstein series defined by

$$
G\left(z, s ; A_{\alpha}, B_{\beta} ; r_{1}, r_{2}\right)=\sum_{m, n=-\infty}^{\infty} \frac{f(\alpha m) f^{*}(\beta n)}{\left(\left(m+r_{1}\right) z+n+r_{2}\right)^{s}}, \operatorname{Re}(s)>2, \operatorname{Im}(z)>0
$$

are investigated for $s=1-r, r \in \mathbb{N}$. Here $\{f(n)\}$ and $\left\{f^{*}(n)\right\},-\infty<n<\infty$ are sequences of complex numbers with period $k>0$, and $A_{\alpha}=\{f(\alpha n)\}$ and $B_{\beta}=\left\{f^{*}(\beta n)\right\}, \alpha, \beta \in \mathbb{Z}$. Appearing in the transformation formulas are generalizations of Dedekind sums involving the periodic Bernoulli function. Reciprocity law is proved for periodic Apostol-Dedekind sum outside of the context of the transformation formulas. Furthermore, transformation formulas are presented for $G\left(z, s ; A_{\alpha}, I ; r_{1}, r_{2}\right)$ and $G\left(z, s ; I, A_{\alpha} ; r_{1}, r_{2}\right)$, where $I=\{1\}$. As an application of these formulas, analogues of Ramanujan's formula for periodic zeta-functions are derived.

Keywords : Eisenstein series, Zeta functions, Dedekind sums, Bernoulli numbers and polynomials.

MSC 2010 : 11M36, 11M41, 11F20, 11B68.
\end{abstract}

\section{Introduction}

For integers $c$ and $d$ with $c>0$, the classical Dedekind sum $s(d, c)$, arising in the transformation formula of the Dedekind eta-function $\eta(z)$, is defined by

$$
s(d, c)=\sum_{n(\bmod c)}\left(\left(\frac{n}{c}\right)\right)\left(\left(\frac{d n}{c}\right)\right)
$$

where the sawtooth function is defined by

$$
((x))= \begin{cases}x-[x]-1 / 2, & \text { if } x \in \mathbb{R} \backslash \mathbb{Z} \\ 0, & \text { if } x \in \mathbb{Z}\end{cases}
$$

with $[x]$ the floor function. One of the most important properties of Dedekind sums is the reciprocity formula

$$
s(d, c)+s(c, d)=-\frac{1}{4}+\frac{1}{12}\left(\frac{d}{c}+\frac{c}{d}+\frac{1}{d c}\right)
$$

whenever $c$ and $d$ are coprime positive integers. For several proofs of (1) and generalizations for Dedekind sums, for example, see $[1,4,5,6,8,9,14,15,16,19,23,24,25,26,27,29]$.

There are several other functions such as Eisenstein series, which possess transformation formula similar to $\log \eta(z)$. Lewittes [22] has discovered a method of obtaining transformation formulas for certain generalized Eisenstein series which developed by Berndt [4]. Berndt's transformation formulas contain transformation formulas previously established by Apostol, C. Meyer, Dieter, Schoeneberg and others. By this way, Berndt obtain transformation formula for several types of 
Eisenstein series involving exponential function and primitive characters $[6,5,8]$. Following Berndt, Meyer [24], Sekine [28] and Lim [23] give transformation formulas for aforementioned classes of functions. Arising in the transformation formulas are various types of Dedekind sums, all of which satisfy reciprocity theorems.

From the principal theorem of [6], Berndt [11] and Goldberg [18] derived a number of transformation formulas for the logarithms of the classical theta-functions in which Dedekind-like sums or Hardy-Berndt sums appear. A character generalization of certain Hardy-Berndt sums have been achieved by Can and Kurt [13] from the main theorem of [5].

One of the most interesting corollaries of [4, Theorem 2] is Ramanujan's formula for $\zeta(2 n+1)$, $n \geq 1$. It is shown in [10] that this and also Euler's formula for $\zeta(2 n), n \geq 1$, are both consequences of [4, Theorem 2]. Moreover, the character analogue of Ramanujan's formula for Dirichlet $L$ function is due to Katayama [20] and is proved by Berndt [8] via transformation formulas. In [12], Bradley has achieved periodic analogues of Ramanujan's formula by employing the partial fraction expansion of the hyperbolic cotangent. Katayama's and Berndt's formulas are immediate consequences of Bradley's theorems.

Recently, the authors [17] have derived transformation formulas for a very large class of Eisenstein series defined by

$$
G\left(z, s ; A_{\alpha}, B_{\beta} ; r_{1}, r_{2}\right)=\sum_{m, n=-\infty}^{\infty} \frac{f(\alpha m) f^{*}(\beta n)}{\left(\left(m+r_{1}\right) z+n+r_{2}\right)^{s}}, \operatorname{Re}(s)>2, \operatorname{Im}(z)>0
$$

where $\{f(n)\}$ and $\left\{f^{*}(n)\right\},-\infty<n<\infty$ are sequences of complex numbers with period $k>0$, and $A_{\alpha}=\{f(\alpha n)\}$ and $B_{\beta}=\left\{f^{*}(\beta n)\right\}, \alpha, \beta \in \mathbb{Z}$. In (2), the dash / means that the possible pair $m=-r_{1}, n=-r_{2}$ is excluded from the summation. These transformation formulas are extensions of the principal theorems of Berndt $[4,5,8]$. Letting $s=0$ in these transformation formulas, generalizations of Dedekind sums (called periodic Dedekind sums) involving the periodic Bernoulli function appear. It is shown that these sums still obey reciprocity laws. In addition, relations between various infinite series and evaluations of several infinite series are deduced from the transformation formulas. Also, periodic Dedekind sums for some special values of $A_{\alpha}$ and $B_{\beta}$ are illustrated.

In the present paper, we wish to investigate transformation formulas for positive integer $r$ and $s=1-r$ in Theorem 2 (see below). Appearing in these transformation formulas are generalizations of Dedekind sums as

$$
\sum_{n=1}^{c k} f^{*}(b n) P_{m}\left(\frac{n}{c k}\right) P_{r+1-m}\left(d n / c, A_{c}\right)
$$

where $P_{r}\left(x, A_{c}\right)$ is the periodic Bernoulli function (see (8)). In Section 3, we prove two reciprocity formulas. The first one is for the function involving periodic Dedekind sums and the second is for periodic Apostol-Dedekind sum. In Section 4, we present transformation formulas which yield analogues of Ramanujan's formula for periodic zeta-functions. Bradley's formulas are consequences of one of our theorems and a very special cases of an infinite class of similar formulas.

\section{Notation and preliminaries}

Let $\mathbb{H}=\{z: \operatorname{Im}(z)>0\}$ denote the upper half-plane and $\mathbb{K}=\{z: \operatorname{Re}(z)>-d / c, \operatorname{Im}(z)>0\}$ denote the upper quarter-plane. We use the modular transformation $V z=V(z)=(a z+b) /(c z+d)$ where $a, b, c$ and $d$ are integers with $a d-b c=1$ and $c>0$. We use the notation $\{x\}$ for the fractional part of $x$, and $\lambda_{x}$ for the characteristic function of integers. We put $e(z)=e^{2 \pi i z}$ and unless otherwise stated, we use the branch of the argument defined by $-\pi \leq \arg z<\pi$.

Let $\{f(n)\}=A,-\infty<n<\infty$ be sequence of complex numbers with period $k>0$. For $|t|<2 \pi / k$, the periodic Bernoulli numbers and polynomials are defined by means of the generating functions [7]

$$
\sum_{n=0}^{k-1} \frac{t f(n) e^{n t}}{e^{k t}-1}=\sum_{j=0}^{\infty} \frac{B_{j}(A)}{j !} t^{j}
$$


and

$$
\sum_{n=0}^{k-1} \frac{t f(-n) e^{(n+x) t}}{e^{k t}-1}=\sum_{j=0}^{\infty} \frac{B_{j}(x, A)}{j !} t^{j} .
$$

Note that, when $A=I=\{1\},(3)$ and (4) reduce to ordinary Bernoulli numbers and polynomials, defined by the generating functions [3]

$$
\begin{aligned}
& \frac{t}{e^{t}-1}=\sum_{n=0}^{\infty} B_{n} \frac{t^{n}}{n !},|t|<2 \pi, \\
& \frac{t e^{x t}}{e^{t}-1}=\sum_{n=0}^{\infty} B_{n}(x) \frac{t^{n}}{n !},|t|<2 \pi,
\end{aligned}
$$

respectively. Notice that $B_{0}(x)=1, B_{1}=-1 / 2, B_{1}(1)=1 / 2, B_{2 n+1}=B_{2 n-1}(1 / 2)=0, n \geq 1$, and $B_{1}(x)=x-1 / 2$. by

Throughout this paper, the $n$-th Bernoulli function will be denoted by $P_{n}(x)$ and is defined

$$
n ! P_{n}(x)=B_{n}(x-[x]) .
$$

In particular $P_{1}(x)=x-[x]-1 / 2$. These functions satisfy the Raabe or multiplication formula for all real $x$

$$
P_{n}(x)=k^{n-1} \sum_{m=0}^{k-1} P_{n}\left(\frac{m+x}{k}\right)
$$

and the reflection identity $P_{n}(-x)=(-1)^{n} P_{n}(x)$ except $n=1$ and $x \in \mathbb{Z}$, in that case

$$
P_{1}(-x)=P_{1}(x)=P_{1}(0)=-1 / 2 .
$$

The periodic Bernoulli functions $P_{r}(x, A)$, are functions with period $k$, may be defined by [7]

$$
P_{0}(x, A)=B_{0}(A)=\frac{1}{k} \sum_{m=0}^{k-1} f(m)
$$

and

$$
P_{r}(x, A)=k^{r-1} \sum_{v=0}^{k-1} f(-v) P_{r}\left(\frac{v+x}{k}\right), r \geq 1
$$

for all real $x$. Also, the periodic Bernoulli function $P_{r}\left(x, A_{c}\right)$ is defined in [17] by

$$
P_{r}\left(x, A_{c}\right)=k^{r-1} \sum_{v=0}^{k-1} f(-c v) P_{r}\left(\frac{v+x}{k}\right) .
$$

Define the sequence $\widehat{A}=\{\widehat{f}(n)\}$ by

$$
\widehat{f}(n)=\frac{1}{k} \sum_{j=0}^{k-1} f(j) e(-n j / k)
$$

for $-n<\infty<n$. These are the finite Fourier series coefficients of $\{f(n)\}$. Clearly $\widehat{A}$ also has period $k$. Note that (9) holds if and only if

$$
f(n)=\sum_{j=0}^{k-1} \widehat{f}(j) e(n j / k), \quad-n<\infty<n .
$$

We need the following Lemma and Theorems given in [17]. 
Lemma 1 Let $z \in \mathbb{H}, \operatorname{Re}(s)>2$ and $\beta \beta^{-1} \equiv 1(\bmod k)$. Then,

$$
\begin{aligned}
& \Gamma(s) G\left(z, s ; A_{\alpha}, B_{\beta} ; r_{1}, r_{2}\right) \\
& =(-2 \pi i / k)^{s} k\left(A\left(z, s ; A_{\alpha}, \widehat{B}_{-\beta^{-1}} ; r_{1}, r_{2}\right)+e(s / 2) A\left(z, s ; A_{-\alpha}, \widehat{B}_{\beta^{-1}} ;-r_{1},-r_{2}\right)\right) \\
& \quad+\lambda_{r_{1}} f\left(-\alpha r_{1}\right) \Gamma(s)\left(L\left(s ; B_{\beta} ; r_{2}\right)+e(s / 2) L\left(s ; B_{-\beta} ;-r_{2}\right)\right)
\end{aligned}
$$

where

$$
A\left(z, s ; A_{\alpha}, A_{\beta} ; r_{1}, r_{2}\right)=\sum_{m>-r_{1}} f(\alpha m) \sum_{n=1}^{\infty} f(\beta n) e\left(n \frac{\left(m+r_{1}\right) z+r_{2}}{k}\right) n^{s-1}
$$

and

$$
L\left(s ; A_{\beta} ; \theta\right)=\sum_{n>-\theta} f(n \beta)(n+\theta)^{-s}, \text { for } \operatorname{Re}(s)>1 \text { and } \theta \text { real. }
$$

Note that since $L\left(s ; B_{\beta} ; r_{2}\right)$ can be analytically continued to the entire $s$-plane with the possible exception $s=1$ and since $A\left(z, s ; A_{\alpha}, B_{\beta} ; r_{1}, r_{2}\right)$ is entire function of $s, G\left(z, s ; A_{\alpha}, B_{\beta} ; r_{1}, r_{2}\right)$ can be analytically continued to the entire $s$-plane with the possible exception $s=1$.

Theorem 2 Define $R_{1}=a r_{1}+c r_{2}$ and $R_{2}=b r_{1}+d r_{2}$, in which $r_{1}$ and $r_{2}$ are arbitrary real numbers. Let $\rho=\rho\left(R_{1}, R_{2}, c, d\right)=\left\{R_{2}\right\} c-\left\{R_{1}\right\} d$. Suppose first that $a \equiv d \equiv 0(\bmod k)$. Then for $z \in \mathbb{K}$ and all $s$,

$$
\begin{aligned}
& (c z+d)^{-s} \Gamma(s) G\left(V z, s ; A, B ; r_{1}, r_{2}\right) \\
& =\Gamma(s) G\left(z, s ; B_{-b}, A_{-c} ; R_{1}, R_{2}\right)-2 i \Gamma(s) \sin (\pi s) L\left(s ; A_{c} ;-R_{2}\right) f^{*}\left(b R_{1}\right) \lambda_{R_{1}} \\
& +e(-s / 2) \sum_{j=1}^{c} \sum_{\mu=0}^{k-1} \sum_{v=0}^{k-1} f\left(c\left(\left[R_{2}+d\left(j-\left\{R_{1}\right\}\right) / c\right]-v\right)\right) f^{*}\left(b\left(\mu c+j+\left[R_{1}\right]\right)\right) \\
& \quad \times I\left(z, s, c, d, r_{1}, r_{2}\right)
\end{aligned}
$$

where $L\left(s ; A_{c} ; R_{2}\right)$ is given by (13) and

$$
\begin{aligned}
& I\left(z, s, c, d, r_{1}, r_{2}\right) \\
& \quad=\int_{C} u^{s-1} \frac{\exp \left(-\left(\left(c \mu+j-\left\{R_{1}\right\}\right) / c k\right)(c z+d) k u\right)}{\exp (-k u(c z+d))-1} \frac{\exp (((v+\{(d j+\rho) / c\}) / k) k u)}{\exp (k u)-1} d u .
\end{aligned}
$$

Here, we choose the branch of $u^{s}$ with $0<\arg u<2 \pi$. Also, $C$ is a loop beginning at $+\infty$, proceeding in the upper half-plane, encircling the origin in the positive direction so that $u=0$ is the only zero of $(\exp (-k u(c z+d))-1)(\exp (k u)-1)$ lying "inside" the loop, and then returning to $+\infty$ in the lower half-plane.

Secondly, if $b \equiv c \equiv 0(\bmod k)$, then for $z \in \mathbb{K}$ and all $s$,

$$
\begin{aligned}
& (c z+d)^{-s} \Gamma(s) G\left(V z, s ; A, B ; r_{1}, r_{2}\right) \\
& =\Gamma(s) G\left(z, s ; A_{d}, B_{a} ; R_{1}, R_{2}\right)-2 i \Gamma(s) \sin (\pi s) L\left(s ; B_{-a} ;-R_{2}\right) f\left(-d R_{1}\right) \lambda_{R_{1}} \\
& \quad+e(-s / 2) \sum_{j=1}^{c} \sum_{\mu=0}^{k-1} \sum_{v=0}^{k-1} f^{*}\left(-a\left(\left[R_{2}+d\left(j-\left\{R_{1}\right\}\right) / c\right]-v+d \mu\right)\right) f\left(-d\left(j+\left[R_{1}\right]\right)\right) I\left(z, s, c, d, r_{1}, r_{2}\right) .
\end{aligned}
$$

Theorem 3 Under the conditions of Theorem 2 , for $a \equiv d \equiv 0(\bmod k)$ we have

$$
\begin{aligned}
& (c z+d)^{-s} \Gamma(s) G\left(V z, s ; B_{-\beta}, A_{-\alpha} ; r_{1}, r_{2}\right) \\
& =\Gamma(s) G\left(z, s ; A_{\alpha b}, B_{\beta c} ; R_{1}, R_{2}\right)-2 i \Gamma(s) \sin (\pi s) f\left(-\alpha b R_{1}\right) L\left(s ; B_{-\beta c} ;-R_{2}\right) \\
& +e(-s / 2) \sum_{j=1}^{c} \sum_{\mu=0}^{k-1} \sum_{v=0}^{k-1} f\left(-\alpha b\left(\mu c+j+\left[R_{1}\right]\right)\right) f^{*}\left(-\beta c\left(\left[R_{2}+d\left(j-\left\{R_{1}\right\}\right) / c\right]-v\right)\right) \\
& \quad \times I\left(z, s, c, d, r_{1}, r_{2}\right),
\end{aligned}
$$

where $I\left(z, s, c, d, r_{1}, r_{2}\right)$ is given by (15).

For simplicity, the function $G\left(z, s ; A_{\alpha}, B_{\beta} ; 0,0\right)$ will be denoted by $G\left(z, s ; A_{\alpha}, B_{\beta}\right)$ and $G\left(z, s ; A_{1}\right.$, $\left.B_{1} ; r_{1}, r_{2}\right)=G\left(z, s ; A, B ; r_{1}, r_{2}\right)$. 


\section{The periodic analogue of Dedekind sum}

Theorem 2 can be simplified when $s=1-r$ is an integer for $r \geq 1$. In [17], we investigate Theorem 2 for the case $r=1$ and $r_{1}, r_{2} \in \mathbb{R}$ in detail. Therefore, in this section we assume that $r>1$ and $r_{1}=r_{2}=0$. In this case, by the residue theorem, $I(z, 1-r, c, d, 0,0)$ becomes with the aid of $(5)$

$$
\begin{aligned}
& I(z, 1-r, c, d, 0,0) \\
& =\frac{2 \pi i k^{r-1}}{(r+1) !} \sum_{m=0}^{r+1}\left(\begin{array}{c}
r+1 \\
m
\end{array}\right)(-(c z+d))^{m-1} B_{m}\left(\frac{c \mu+j}{c k}\right) B_{r+1-m}\left(\frac{v+\{d j / c\}}{k}\right) .
\end{aligned}
$$

Let $a \equiv d \equiv 0(\bmod k)$. Substituting $(18)$ in $(14)$ gives

$$
\begin{aligned}
& \lim _{s \rightarrow 1-r}\left((c z+d)^{-s} \Gamma(s) G(V z, s ; A, B)-\Gamma(s) G\left(z, s ; B_{-b}, A_{-c}\right)\right) \\
& =-2 i f^{*}(0) \lim _{s \rightarrow 1-r} \Gamma(s) \sin (\pi s) L\left(s, A_{c} ; 0\right) \\
& \quad+(-1)^{r-1} \frac{2 \pi i k^{r-1}}{(r+1) !} \sum_{m=0}^{r+1}\left(\begin{array}{c}
r+1 \\
m
\end{array}\right)(-(c z+d))^{m-1} \\
& \quad \times \sum_{j=1}^{c} \sum_{\mu=0}^{k-1} \sum_{v=0}^{k-1} f(c([d j / c]-v)) f^{*}(b(\mu c+j)) B_{m}\left(\frac{c \mu+j}{c k}\right) B_{r+1-m}\left(\frac{v+\{d j / c\}}{k}\right) .
\end{aligned}
$$

We must evaluate the triple sum in (19). We first note that the triple sum is invariant by replacing $B_{m}\left(\frac{c \mu+j}{c k}\right)$ by $m ! P_{m}\left(\frac{c \mu+j}{c k}\right)$ for $1 \leq j \leq c-1$ and $B_{r+1-m}\left(\frac{v+\{d j / c\}}{k}\right)$ by $(r+1-m) ! P_{r+1-m}\left(\frac{v+\{d j / c\}}{k}\right)$. Also replacing $v-[d j / c]$ by $v$ yields

$$
\begin{aligned}
& \sum_{j=1}^{c} \sum_{\mu=0}^{k-1} \sum_{v=0}^{k-1} f(c([d j / c]-v)) f^{*}(b(\mu c+j)) B_{m}\left(\frac{c \mu+j}{c k}\right) B_{r+1-m}\left(\frac{v+\{d j / c\}}{k}\right) \\
& \left.=m !(r+1-m) ! \sum_{j=1}^{c-1} \sum_{\mu=0}^{k-1} f^{*}(b(\mu c+j)) P_{m}\left(\frac{c \mu+j}{c k}\right) \sum_{v=0}^{k-1} f(-c v)\right) P_{r+1-m}\left(\frac{v+d j / c}{k}\right) \\
& \quad+(r+1-m) ! \sum_{\mu=0}^{k-1} f^{*}(b c(\mu+1)) B_{m}\left(\frac{\mu+1}{k}\right) \sum_{v=0}^{k-1} f(-c v) P_{r+1-m}\left(\frac{v}{k}\right) .
\end{aligned}
$$

Here adding and subtracting the term $j=c$ and writing $\mu c+j=n$, the right-hand side becomes

$$
\begin{aligned}
& m !(r+1-m) ! k^{m-r} \sum_{n=1}^{c k} f^{*}(b n) P_{m}\left(\frac{n}{c k}\right) P_{r+1-m}\left(\frac{d n}{c}, A_{c}\right) \\
& \quad+(r+1-m) ! k^{m-r} f^{*}(0) P_{r+1-m}\left(0, A_{c}\right)\left(B_{m}(1)-m ! P_{m}(0)\right) \\
& =m !(r+1-m) ! k^{m-r} \sum_{n=1}^{c k} f^{*}(b n) P_{m}\left(\frac{n}{c k}\right) P_{r+1-m}\left(\frac{d n}{c}, A_{c}\right) \\
& \quad+r ! k^{1-r} f^{*}(0) P_{r}\left(0, A_{c}\right) .
\end{aligned}
$$

Then, we can give the following definition.

Definition 4 Let $a d-b c=1$ and $a \equiv d \equiv 0(\bmod k)$. The periodic Dedekind sum $s_{r+1-m, m}\left(d, c ; B_{b} ; A_{c}\right)$ is defined by

$$
s_{r+1-m, m}\left(d, c ; B_{b} ; A_{c}\right)=\sum_{n=1}^{c k} f^{*}(b n) P_{m}\left(\frac{n}{c k}\right) P_{r+1-m}\left(d n / c, A_{c}\right) .
$$


On the other hand, using the Euler's reflection formula $\Gamma(s) \Gamma(1-s)=\pi / \sin (\pi s)$ and the fact [7, Corollary 6.4 for $a=0]$, we can write

$$
\lim _{s \rightarrow 1-r} \Gamma(s) \sin (\pi s) L\left(s, A_{c} ; 0\right)=\pi(-1)^{r-1} P_{r}\left(0, A_{c}\right) .
$$

Thus, we have

$$
\begin{aligned}
& \lim _{s \rightarrow 1-r} \Gamma(s)\left((c z+d)^{-s} G(V z, s ; A, B)-G\left(z, s ; B_{-b}, A_{-c}\right)\right) \\
& =(-1)^{r-1} 2 \pi i \sum_{m=0}^{r+1} k^{m-1}(-(c z+d))^{m-1} s_{r+1-m, m}\left(d, c ; B_{b} ; A_{c}\right) .
\end{aligned}
$$

For $b \equiv c \equiv 0(\bmod k)$, by similar arguments, one can hold

$$
\begin{aligned}
& \lim _{s \rightarrow 1-r} \Gamma(s)\left((c z+d)^{-s} G(V z, s ; A, B)-G\left(z, s ; A_{d}, B_{a}\right)\right) \\
& =(-1)^{r-1} 2 \pi i \sum_{m=0}^{r+1} k^{m-1}(-(c z+d))^{m-1} \sum_{n=1}^{c k} f(-d n) P_{m}\left(\frac{n}{c k}\right) P_{r+1-m}\left(d n / c, B_{-a}\right) .
\end{aligned}
$$

Definition 5 Let $a d-b c=1$ and $b \equiv c \equiv 0(\bmod k)$. The periodic Dedekind sum $s_{r+1-m, m}\left(d, c ; A_{-d} ; B_{-a}\right)$ is defined by

$$
s_{r+1-m, m}\left(d, c ; A_{-d} ; B_{-a}\right)=\sum_{n=1}^{c k} f(-d n) P_{m}\left(\frac{n}{c k}\right) P_{r+1-m}\left(d n / c, B_{-a}\right) .
$$

We summarize the results obtained above in the next theorem.

Theorem 6 Let $r>1$ be an integer and $z \in \mathbb{H}$. If $a \equiv d \equiv 0(\bmod k)$, then

$$
\begin{aligned}
& \lim _{s \rightarrow 1-r} \Gamma(s)\left((c z+d)^{-s} G(V z, s ; A, B)-G\left(z, s ; B_{-b}, A_{-c}\right)\right) \\
& =2 \pi i(-1)^{r-1} \sum_{m=0}^{r+1} k^{m-1}(-(c z+d))^{m-1} s_{r+1-m, m}\left(d, c ; B_{b} ; A_{c}\right) .
\end{aligned}
$$

If $b \equiv c \equiv 0(\bmod k)$, then

$$
\begin{aligned}
& \lim _{s \rightarrow 1-r} \Gamma(s)\left((c z+d)^{-s} G(V z, s ; A, B)-G\left(z, s ; A_{d}, B_{a}\right)\right) \\
& =2 \pi i(-1)^{r-1} \sum_{m=0}^{r+1} k^{m-1}(-(c z+d))^{m-1} s_{r+1-m, m}\left(d, c ; A_{-d} ; B_{-a}\right) .
\end{aligned}
$$

\subsection{Reciprocity Theorems}

In this section, we prove two reciprocity theorems. The first one can be viewed as the reciprocity formula for the function $F\left(d, c, z ; r ; A_{c} ; B_{b}\right)$ given by

$$
F\left(d, c, z ; r ; A_{c} ; B_{b}\right)=\sum_{m=0}^{r+1} k^{m-1}(-(d z+c))^{m-1} s_{r+1-m, m}\left(c, d ; A_{c} ; B_{b}\right)
$$

The second one is the reciprocity formula for periodic Apostol-Dedekind sum $s_{r}\left(d, c ; A_{\alpha} ; B_{\beta}\right)$, defined by

$$
s_{r}\left(d, c ; A_{\alpha} ; B_{\beta}\right)=\sum_{n=1}^{c k} f(\alpha n) P_{1}\left(\frac{n}{c k}\right) P_{r}\left(d n / c, B_{\beta}\right) .
$$


Theorem 7 Let $a d-b c=1$ with $d, c>0$ and $r>1$ be an integer. For $a \equiv d \equiv 0(\bmod k)$ and $z \in \mathbb{C}-\{0, c / d\}$, we have

$$
\begin{aligned}
& F\left(d,-c, z ; r ; A_{c} ; B_{-b}\right)-z^{r-1} F\left(c, d,-\frac{1}{z} ; r ; B_{b} ; A_{c}\right) \\
& =\sum_{m=0}^{r+1}(-z)^{m-1} P_{m}\left(0, A_{-c}\right) P_{r+1-m}\left(0, B_{-b}\right),
\end{aligned}
$$

where $F\left(d, c, z ; r ; A_{c} ; B_{b}\right)$ is defined by (23).

Proof. Replacing $z$ by $-1 / z$ in $(21)$ gives

$$
\begin{aligned}
& \lim _{s \rightarrow 1-r} \Gamma(s) z^{s}\left(\frac{1}{(d z-c)^{s}} G(T z, s ; A, B)-z^{-s} G\left(-\frac{1}{z}, s ; B_{-b}, A_{-c}\right)\right) \\
& =(-1)^{r-1} 2 \pi i \sum_{m=0}^{r+1} k^{m-1}\left(-\left(\frac{d z-c}{z}\right)\right)^{m-1} s_{r+1-m, m}\left(d, c ; B_{b} ; A_{c}\right)
\end{aligned}
$$

and replacing $V z$ by $T z=(b z-a) /(d z-c)=V(-1 / z)$ in $(22)$ gives

$$
\begin{aligned}
& \lim _{s \rightarrow 1-r} \Gamma(s)\left(\frac{1}{(d z-c)^{s}} G(T z, s ; A, B)-G\left(z, s ; A_{-c}, B_{b}\right)\right) \\
& =(-1)^{r-1} 2 \pi i \sum_{m=0}^{r+1} k^{m-1}(-(d z-c))^{m-1} s_{r+1-m, m}\left(-c, d ; A_{c} ; B_{-b}\right) .
\end{aligned}
$$

Since

$$
\begin{aligned}
& \lim _{s \rightarrow 1-r} z^{s} \Gamma(s)\left(\frac{1}{(d z-c)^{s}} G(T z, s ; A, B)-G\left(z, s ; A_{-c}, B_{b}\right)\right) \\
& =\lim _{s \rightarrow 1-r} z^{s} \Gamma(s)\left(\frac{1}{(d z-c)^{s}} G(V(-1 / z), s ; A, B)-\frac{1}{z^{s}} G\left(-1 / z, s ; B_{-b}, A_{-c}\right)\right) \\
& \quad+\lim _{s \rightarrow 1-r} z^{s} \Gamma(s)\left(\frac{1}{z^{s}} G\left(-1 / z, s ; B_{-b}, A_{-c}\right)-G\left(z, s ; A_{-c}, B_{b}\right)\right),
\end{aligned}
$$

it is sufficient to evaluate the following limit in order to prove the reciprocity formula

$$
\lim _{s \rightarrow 1-r} z^{s} \Gamma(s)\left(\frac{1}{z^{s}} G\left(-1 / z, s ; B_{-b}, A_{-c}\right)-G\left(z, s ; A_{-c}, B_{b}\right)\right) .
$$

For this, taking $V z=-1 / z$ in (17) and using (18) we have

$$
\begin{aligned}
\lim _{s \rightarrow 1-r} \Gamma(s)\left(z^{-s} G\left(-1 / z, s ; B_{-b}, A_{-c}\right)-G\left(z, s ; A_{-c}, B_{b}\right)\right) \\
=-2 \pi i f(0)(-1)^{r-1} P_{r}\left(0, B_{-b}\right) \\
\quad+\frac{2 \pi i(-k)^{r-1}}{(r+1) !} \sum_{m=0}^{r+1}\left(\begin{array}{c}
r+1 \\
m
\end{array}\right)(-z)^{m-1} \sum_{\mu=0}^{k-1} f(c(\mu+1)) B_{m}\left(\frac{\mu+1}{k}\right) \sum_{v=0}^{k-1} f^{*}(b v) B_{r+1-m}\left(\frac{v}{k}\right) .
\end{aligned}
$$

It is seen from (8) that

$$
\begin{aligned}
& \sum_{v=0}^{k-1} f^{*}(b v) B_{r+1-m}\left(\frac{v}{k}\right)\left(\sum_{\mu=1}^{k-1} f(c \mu) B_{m}\left(\frac{\mu}{k}\right)+f(0) B_{m}(1)\right) \\
& =(r+1-m) ! k^{m-r} P_{r+1-m}\left(0, B_{-b}\right)\left(m ! k^{1-m} P_{m}\left(0, A_{-c}\right)+\left\{\begin{array}{ll}
f(0), & \text { if } m=1 ; \\
0, & \text { otherwise }
\end{array}\right) .\right.
\end{aligned}
$$


Substituting these in (27) and using (20) yield

$$
\begin{aligned}
& \lim _{s \rightarrow 1-r} \Gamma(s)\left(\frac{1}{z^{s}} G\left(-1 / z, s ; B_{-b}, A_{-c}\right)-G\left(z, s ; A_{-c}, B_{b}\right)\right) \\
& =2 \pi i(-1)^{r-1} \sum_{m=0}^{r+1}(-z)^{m-1} P_{m}\left(0, A_{-c}\right) P_{r+1-m}\left(0, B_{-b}\right) .
\end{aligned}
$$

Thus, combining (28), (25) and (26) gives

$$
\begin{aligned}
& F\left(d,-c, z ; r ; A_{c} ; B_{-b}\right)-z^{r-1} F\left(c, d,-\frac{1}{z} ; r ; B_{b} ; A_{c}\right) \\
& =\sum_{m=0}^{r+1}(-z)^{m-1} P_{m}\left(0, A_{-c}\right) P_{r+1-m}\left(0, B_{-b}\right),
\end{aligned}
$$

for $z \in \mathbb{H}$. By analytic continuation, (29) is valid for $z \in \mathbb{C}-\{0, c / d\}$.

Before stating and proving a reciprocity formula for the periodic Apostol-Dedekind sum $s_{r}\left(d, c ; A_{\alpha} ; B_{\beta}\right)$ given by (24), we discuss this sum.

Assume that $\alpha \equiv 0(\bmod k)$. Since $A_{\alpha}=\{f(0)\}=f(0) I$, it follows that

$$
\begin{aligned}
& s_{r}\left(d, c ; A_{\alpha} ; B_{\beta}\right)=f(0) \sum_{n=1}^{c k} P_{1}\left(\frac{n}{c k}\right) P_{r}\left(d n / c, B_{\beta}\right), \\
& s_{r}\left(d, c ; B_{\beta} ; A_{\alpha}\right)=f(0) \sum_{n=1}^{c k} f^{*}(\beta n) P_{1}\left(\frac{n}{c k}\right) P_{r}\left(\frac{d n}{c}\right),
\end{aligned}
$$

which are periodic extensions of Berndt's character Dedekind sums $S_{2}(d, c ; \chi)$ and $S_{1}(d, c ; \chi)$ defined in [8, Eqs. (6.2) and (6.1)], respectively.

If $\alpha \equiv \beta \equiv 0(\bmod k)$, then the sum $s_{r}\left(d, c ; A_{\alpha} ; B_{\beta}\right)$ is equal to $f(0) f^{*}(0) s_{r}(d, c)$, where $s_{r}(d, c)$ is the Apostol-Dedekind sum

$$
s_{r}(d, c)=\sum_{j=0}^{c-1} P_{1}\left(\frac{j}{c}\right) P_{r}\left(\frac{d j}{c}\right) .
$$

In general, writing $v+j k, 1 \leq v \leq k, 0 \leq j<c$ in place of $n$ in the definition of $s_{r}\left(d, c ; A_{\alpha} ; B_{\beta}\right)$ and using (8), we have

$$
\begin{aligned}
s_{r}\left(d, c ; A_{\alpha} ; B_{\beta}\right) & =\sum_{n=1}^{c k} f(\alpha n) P_{1}\left(\frac{n}{c k}\right) P_{r}\left(d n / c, B_{\beta}\right) \\
& =k^{r-1} \sum_{v=1}^{k} \sum_{j=0}^{c-1} f(\alpha v) P_{1}\left(\frac{j+\frac{v}{k}}{c}\right) \sum_{\mu=1}^{k} f^{*}(-\beta \mu) P_{r}\left(\frac{d\left(j+\frac{v}{k}\right)}{c}+\frac{\mu}{k}\right) \\
& =k^{r-1} \sum_{v=1}^{k} \sum_{\mu=1}^{k} f(\alpha v) f^{*}(-\beta \mu) \sum_{j=0}^{c-1} P_{1}\left(\frac{j+\frac{v}{k}}{c}\right) P_{r}\left(\frac{d\left(j+\frac{v}{k}\right)}{c}+\frac{\mu}{k}\right) .
\end{aligned}
$$

Observe that the sum over $j$ is the generalized Dedekind sums due to Carlitz [14] (or Takacs [29]) given by

$$
s_{r}(d, c \mid x, y)=\sum_{j=0}^{c-1} \bar{B}_{1}\left(\frac{j+y}{c}\right) \bar{B}_{r}\left(\frac{d(j+y)}{c}+x\right)
$$

where $\bar{B}_{r}(x)=r ! P_{r}(x)$. Then

$$
s_{r}\left(d, c ; A_{\alpha} ; B_{\beta}\right)=\frac{k^{r-1}}{r !} \sum_{v=1}^{k} \sum_{\mu=1}^{k} f(\alpha v) f^{*}(-\beta \mu) s_{r}\left(d, c \mid \frac{\mu}{k}, \frac{v}{k}\right) .
$$


This shows that we may achieve a reciprocity formula for $s_{r}\left(d, c ; A_{\alpha} ; B_{\beta}\right)$ with the help of the following reciprocity law for $s_{r}(d, c \mid x, y)$ : Let $c$ and $d$ be positive coprime integers. For integers $r \geq 0$ and for real numbers $x$ and $y$, the reciprocity formula holds $[14,29]$

$$
\begin{aligned}
& (r+1)\left[d c^{r} s_{r}(d, c \mid x, y)+c d^{r} s_{r}(c, d \mid y, x)\right] \\
& =\sum_{j=0}^{r+1}\left(\begin{array}{c}
r+1 \\
j
\end{array}\right) c^{j} d^{r+1-j} \bar{B}_{j}(x) \bar{B}_{r+1-j}(y)+r \bar{B}_{r+1}(d y+c x) .
\end{aligned}
$$

Theorem 8 Let $c$ and $d$ be coprime positive integers. For $\alpha, \beta \in \mathbb{Z}$ and $r=0,1,2, \ldots$ the following reciprocity formula holds:

$$
\begin{aligned}
& d c^{r} s_{r}\left(d, c ; A_{-\alpha} ; B_{\beta}\right)+c d^{r} s_{r}\left(c, d ; B_{-\beta} ; A_{\alpha}\right) \\
& =\sum_{j=0}^{r+1} c^{j} d^{r+1-j} P_{r+1-j}\left(0, A_{\alpha}\right) P_{j}\left(0, B_{\beta}\right)+r k^{r-1} \sum_{v=1}^{k} \sum_{\mu=1}^{k} f(-\alpha v) f^{*}(-\beta \mu) P_{r+1}\left(\frac{d v+c \mu}{k}\right) .
\end{aligned}
$$

Proof. From (30), we have

$$
\begin{aligned}
& s_{r}\left(d, c ; A_{-\alpha} ; B_{\beta}\right)=\frac{k^{r-1}}{r !} \sum_{v=1}^{k} \sum_{\mu=1}^{k} f(-\alpha v) f^{*}(-\beta \mu) s_{r}\left(d, c \mid \frac{\mu}{k}, \frac{v}{k}\right), \\
& s_{r}\left(c, d ; B_{-\beta} ; A_{\alpha}\right)=\frac{k^{r-1}}{r !} \sum_{v=1}^{k} \sum_{\mu=1}^{k} f(-\alpha v) f^{*}(-\beta \mu) s_{r}\left(c, d \mid \frac{v}{k}, \frac{\mu}{k}\right)
\end{aligned}
$$

and thus

$$
\begin{aligned}
& (r+1)\left[d c^{r} s_{r}\left(d, c ; A_{-\alpha} ; B_{\beta}\right)+c d^{r} s_{r}\left(c, d ; B_{-\beta} ; A_{\alpha}\right)\right] \\
& =\frac{k^{r-1}}{r !} \sum_{v=1}^{k} \sum_{\mu=1}^{k} f(-\alpha v) f^{*}(-\beta \mu)(r+1)\left[d c^{r} s_{r}\left(d, c \mid \frac{\mu}{k}, \frac{v}{k}\right)+c d^{r} s_{r}\left(c, d \mid \frac{v}{k}, \frac{\mu}{k}\right)\right] .
\end{aligned}
$$

Using the reciprocity formula given by (31) and then (8), the desired result follows.

\section{Values of periodic zeta-functions}

This section is devoted to derive periodic analogues of Ramanujan's formula for $\zeta(2 N+1)$. We accomplish this by applying Theorem 2 and (11), motivated by [8, 10].

For $z \in \mathbb{H}$ and $s$ complex, we recall the special cases of (12) as

$$
\begin{aligned}
& A\left(z, s ; A_{\alpha}, I ; r_{1}, r_{2}\right)=\sum_{m>-r_{1}} f(\alpha m) \sum_{n=1}^{\infty} e\left(n \frac{\left(m+r_{1}\right) z+r_{2}}{k}\right) n^{s-1}, \\
& A\left(z, s ; I, A_{\beta} ; r_{1}, r_{2}\right)=\sum_{m>-r_{1}} \sum_{n=1}^{\infty} f(\beta n) e\left(n \frac{\left(m+r_{1}\right) z+r_{2}}{k}\right) n^{s-1}
\end{aligned}
$$

Let

$$
H\left(z, s ; A_{\alpha}, B_{\beta} ; r_{1}, r_{2}\right)=A\left(z, s ; A_{\alpha}, B_{-\beta} ; r_{1}, r_{2}\right)+e(s / 2) A\left(z, s ; A_{-\alpha}, B_{\beta} ;-r_{1},-r_{2}\right)
$$

and

$$
L_{ \pm}\left(s ; A_{\beta} ; \theta\right)=L\left(s ; A_{\beta} ; \theta\right)+e( \pm s / 2) L\left(s ; A_{-\beta} ;-\theta\right)
$$

where $L\left(s ; A_{\beta} ; \theta\right)$ is given by (13). In particular, let

$$
Z(s, \theta)=L(s ; I ; \theta)=\sum_{n>-\theta}(n+\theta)^{-s}, \text { for } \operatorname{Re}(s)>1 \text { and } \theta \text { real }
$$


and

$H\left(z, s ; A, B ; r_{1}, r_{2}\right)=H\left(z, s ; A_{1}, B_{1} ; r_{1}, r_{2}\right)$ and $A\left(z, s ; A_{\alpha}, B_{-\beta}\right)=A\left(z, s ; A_{\alpha}, B_{-\beta} ; 0,0\right)$.

For non-negative integers $j$ and $\mu$ and for $z \in \mathbb{K}$, define

$$
\begin{aligned}
& I^{*}\left(z, s, c, d, r_{1}, r_{2}\right) \\
& \quad=\int_{C} u^{s-1} \frac{\exp \left(-\left(\left(c \mu+j-\left\{R_{1}\right\}\right) / c k\right)(c z+d) k u\right)}{\exp (-k u(c z+d))-1} \frac{\exp (\{(d j+\rho) / c\} u)}{\exp (u)-1} d u .
\end{aligned}
$$

If $s=-N$, where $N$ is a non-negative integer, (15) and (35) can be calculated by residue theorem

$$
\begin{aligned}
& I\left(z,-N, c, d, r_{1}, r_{2}\right) \\
& =2 \pi i k^{N} \sum_{m+n=N+2} B_{m}\left(\frac{c \mu+j-\left\{R_{1}\right\}}{c k}\right) B_{n}\left(\frac{v+\{(d j+\rho) / c\}}{k}\right) \frac{(-(c z+d))^{m-1}}{m ! n !}
\end{aligned}
$$

and

$$
\begin{aligned}
& I^{*}\left(z,-N, c, d, r_{1}, r_{2}\right) \\
& =2 \pi i k^{N} \sum_{m+n=N+2} B_{m}\left(\frac{c \mu+j-\left\{R_{1}\right\}}{c k}\right) B_{n}(\{(d j+\rho) / c\}) k^{m-1} \frac{(-(c z+d))^{m-1}}{m ! n !}
\end{aligned}
$$

respectively.

Now we state the transformation formulas involving $H\left(z, s ; A, I ; r_{1}, r_{2}\right)$ and $H\left(z, s ; I, A ; r_{1}, r_{2}\right)$.

Theorem 9 Let $z \in \mathbb{K}$ and suppose that $s$ is an arbitrary complex number. If $a \equiv 0(\bmod k)$, then

$$
\begin{aligned}
& (c z+d)^{-s}(-2 \pi i / k)^{s} k H\left(V z, s ; I, \widehat{B} ; r_{1}, r_{2}\right)+\lambda_{r_{1}}(c z+d)^{-s} \Gamma(s) L_{+}\left(s ; B ; r_{2}\right) \\
& =(-2 \pi i / k)^{s} H\left(z, s ; B_{-b}, I ; R_{1}, R_{2}\right)+\lambda_{R_{1}} f^{*}\left(b R_{1}\right) \Gamma(s) Z_{-}\left(s, R_{2}\right) \\
& +e(-s / 2) \sum_{j=1}^{c} \sum_{\mu=0}^{k-1} f^{*}\left(b\left(c \mu+j+\left[R_{1}\right]\right)\right) I^{*}\left(z, s, c, d, r_{1}, r_{2}\right) .
\end{aligned}
$$

If $b \equiv 0(\bmod k)$, then

$$
\begin{aligned}
& (c z+d)^{-s}(-2 \pi i / k)^{s} k H\left(V z, s ; I, \widehat{B} ; r_{1}, r_{2}\right)+\lambda_{r_{1}}(c z+d)^{-s} \Gamma(s) L_{+}\left(s, B, r_{2}\right) \\
& =(-2 \pi i / k)^{s} k H\left(z, s ; I, \widehat{B}_{a} ; R_{1}, R_{2}\right)+\lambda_{R_{1}} \Gamma(s) L_{-}\left(s ; B_{a} ; R_{2}\right) \\
& +e(-s / 2) \sum_{j=1}^{c} \sum_{\mu=0}^{k-1} \sum_{v=0}^{k-1} f^{*}\left(-a\left(\left[R_{2}+d\left(j-\left\{R_{1}\right\}\right) / c\right]-v+d \mu\right)\right) I\left(z, s, c, d, r_{1}, r_{2}\right) .
\end{aligned}
$$

If $c \equiv 0(\bmod k)$, then

$$
\begin{aligned}
& (c z+d)^{-s}(-2 \pi i / k)^{s} H\left(V z, s ; A, I ; r_{1}, r_{2}\right)+\lambda_{r_{1}} f\left(-r_{1}\right)(c z+d)^{-s} \Gamma(s) Z_{+}\left(s, r_{2}\right) \\
& =(-2 \pi i / k)^{s} H\left(z, s ; A_{d}, I ; R_{1}, R_{2}\right)+\lambda_{R_{1}} f\left(-d R_{1}\right) \Gamma(s) Z_{-}\left(s, R_{2}\right) \\
& +e(-s / 2) \sum_{j=1}^{c} \sum_{\mu=0}^{k-1} f\left(-d\left(c \mu+j+\left[R_{1}\right]\right)\right) I^{*}\left(z, s, c, d, r_{1}, r_{2}\right) .
\end{aligned}
$$

If $d \equiv 0(\bmod k)$, then

$$
\begin{aligned}
& (c z+d)^{-s}(-2 \pi i / k)^{s} H\left(V z, s ; A, I ; r_{1}, r_{2}\right)+\lambda_{r_{1}} f\left(-r_{1}\right)(c z+d)^{-s} \Gamma(s) Z_{+}\left(s, r_{2}\right) \\
& =(-2 \pi i / k)^{s} k H\left(z, s ; I, \widehat{A}_{-c} ; R_{1}, R_{2}\right)+\lambda_{R_{1}} \Gamma(s) L_{-}\left(s ; A_{-c} ; R_{2}\right) \\
& +e(-s / 2) \sum_{j=1}^{c} \sum_{\mu=0}^{k-1} \sum_{v=0}^{k-1} f\left(c\left(\left[R_{2}+d\left(j-\left\{R_{1}\right\}\right) / c\right]-v\right)\right) I\left(z, s, c, d, r_{1}, r_{2}\right) .
\end{aligned}
$$


Furthermore, if $s=-N$ is non-negative integer, upon the evaluation of $I\left(z, s, c, d, r_{1}, r_{2}\right)$ and $I^{*}\left(z, s, c, d, r_{1}, r_{2}\right)$ by (36) and (37), respectively, (38)-(41) are valid for $z \in \mathbb{H}$.

Proof. For $z \in \mathbb{K}, \operatorname{Re}(s)>2, M=m a+n c$ and $N=m b+n d$, we have

$$
\begin{aligned}
& G\left(V z, s ; I, B ; r_{1}, r_{2}\right) \\
& =\sum_{M, N=-\infty}^{\infty} f^{*}(N a-M b)\left\{\frac{\left(\left(M+R_{1}\right) z+N+R_{2}\right)}{c z+d}\right\}^{-s} \\
& =\sum_{m, n=-\infty}^{\infty} f^{*}(-m b)\left\{\frac{\left(\left(m+R_{1}\right) z+n+R_{2}\right)}{c z+d}\right\}^{-s}, a \equiv 0(\bmod k), \\
& =\sum_{m, n=-\infty}^{\infty} f^{*}(\text { an })\left\{\frac{\left(\left(m+R_{1}\right) z+n+R_{2}\right)}{c z+d}\right\}^{-s}, b \equiv 0(\bmod k) .
\end{aligned}
$$

To prove (38) and (39), we follow precisely the method in the proof of [17, Theorem 1] then use Lemma 1 and Eqs. (32)-(34). To prove (40) and (41), follow the method outlined above, but begin by examining $G\left(V z, s ; A, I ; r_{1}, r_{2}\right)$ instead of $G\left(V z, s ; I, B ; r_{1}, r_{2}\right)$.

We mention that the character versions of this theorem are given by Berndt [10, Theorem 4.1] for primitive characters $f=\chi_{1}$ and $f^{*}=\chi_{2}$, and in [8, Theorem 3] for $\chi_{1}=\chi=\bar{\chi}_{2}$.

By letting $s=-N$ be a non-positive integer in Theorem 3 of [8], Berndt obtain some interesting formulas for Dirichlet $L$-functions or curious arithmetical results that are the character analogues of Ramanujan's formula for $\zeta(2 N+1)$ via transformation formulas. Periodic analogues of these formulas are due to Bradley [12] and are special cases of Theorem 9.

Theorem 10 (Bradley [12]) Let $N$ be positive integer and let $\gamma$ be an arbitrary positive number.

If $f$ is an even function, then

$$
\begin{aligned}
\zeta & (2 N+1, \widehat{A})-f(0)\left(i \gamma k^{2}\right)^{-2 N} \zeta(2 N+1) \\
= & 2(i \gamma k)^{-2 N} A(i k \gamma,-2 N ; A, I)-2 k A\left(\frac{i}{k \gamma},-2 N ; I, \widehat{A}\right) \\
& +\frac{(2 \pi i)^{2 N+1}}{k^{2 N}} \sum_{m=0}^{N+1} P_{2 m}(0) P_{2 N+2-2 m}(0, A)(i / k \gamma)^{2 m-1},
\end{aligned}
$$

where $\zeta(s, A)=L(s ; A ; 0)$ is the periodic zeta-function [7, Sec. 6]. If $f(0)=0$, (42) is also valid for $N=0$.

If $f$ is an odd function, then

$$
\begin{aligned}
\zeta(2 N, \widehat{A})= & -2(i k \gamma)^{1-2 N} A(i k \gamma, 1-2 N ; A, I)+2 k A\left(\frac{i}{k \gamma}, 1-2 N ; I, \widehat{A}\right) \\
& +\frac{(2 \pi i)^{2 N}}{k^{2 N-1}} \sum_{m=0}^{N} P_{2 m}(0) P_{2 N+1-2 m}(0, A)(i / k \gamma)^{2 m-1} .
\end{aligned}
$$

Proof. Using the functional equation of $\zeta(s, A)$ ([7, Corollary 6.5]), we have

$$
\lim _{s \rightarrow-N} \Gamma(s) L_{-}\left(s ; A_{c} ; 0\right)=e^{-\pi i N / 2}(k / 2 \pi)^{N} \zeta\left(N+1, \widehat{A}_{c}\right) .
$$

Suppose that $f$ is even. Using (36) and (32), equation (41) can be written as

$$
\begin{aligned}
& (c z+d)^{N}(-2 \pi i / k)^{-N}(1+e(-N / 2)) A(V z,-N ; A, I)+f(0) \lim _{s \rightarrow-N}(c z+d)^{-s} \Gamma(s) Z_{+}(s, 0) \\
& =(-2 \pi i / k)^{-N} k(1+e(-N / 2)) A\left(z,-N ; I, \widehat{A}_{-c}\right)+\lim _{s \rightarrow-N} \Gamma(s) L_{-}\left(s ; A_{-c} ; 0\right) \\
& +2 \pi i \frac{(-k)^{N}}{(N+2) !} \sum_{j=1}^{c} \sum_{\mu=0}^{k-1} \sum_{v=0}^{k-1} \sum_{m=0}^{N+2}\left(\begin{array}{c}
N+2 \\
m
\end{array}\right) f(c([d j / c]-v)) \\
& \quad \times B_{m}\left(\frac{c \mu+j}{c k}\right) B_{N+2-m}\left(\frac{v+\{d j / c\}}{k}\right)(-(c z+d))^{m-1} .
\end{aligned}
$$


Replace $N$ by $2 N$, let $V z=-1 / z$ and put $z=i / k \gamma, \gamma>0$. From the facts (7), (8) and (44), we have

$$
\begin{aligned}
& 2(2 \pi \gamma)^{-2 N} A(i k \gamma,-2 N ; A, I)+f(0)(2 \pi k \gamma)^{-2 N} \zeta(2 N+1) \\
& =2(2 \pi i / k)^{-2 N} k A\left(\frac{i}{k \gamma},-2 N ; I, \widehat{A}\right)+(k / 2 \pi)^{2 N}(-1)^{N} \zeta\left(2 N+1, \widehat{A}_{-1}\right) \\
& +2 \pi i \sum_{m=0}^{2 N+2}(-1)^{m} P_{m}(0) P_{2 N+2-m}(0, A)(-i / k \gamma)^{m-1},
\end{aligned}
$$

where we have used that

$$
\sum_{\mu=0}^{k-1} B_{m}\left(\frac{\mu+1}{k}\right)=k^{1-m}(-1)^{m} B_{m}(0) .
$$

Since $P_{2 N+2-m}(0, A) P_{m}(0)=0$ for odd $m$ and even $f$, we can replace $m$ by $2 m$ on the right-hand side of (45), which completes the proof of (42).

Similarly, if $f$ is an odd function, then replacing $N$ by $2 N-1$ and proceeding as in the proof of (42) give

$$
\begin{aligned}
& 2(i k \gamma)^{1-2 N} A(i k \gamma, 1-2 N ; A, I)-2 k A\left(\frac{i}{k \gamma}, 1-2 N ; I, \widehat{A}\right) \\
& =\zeta\left(2 N, \widehat{A}_{-1}\right)+\frac{(2 \pi i)^{2 N}}{k^{2 N-1}} \sum_{m=0}^{2 N+1} P_{2 N+1-m}(0, A) P_{m}(0)(i / k \gamma)^{m-1},
\end{aligned}
$$

which completes the proof of (43) by replacing $m$ by $2 m$ on the right-hand side of (46).

Note that Theorem 10 is just one of an infinite class of such formulas that can be deduced from Theorem 9 when $s=-N$ and $r_{1}=r_{2}=0$. Similar formulas for $\zeta(2 N+1, B)$ and $\zeta(2 N, B)$ can be obtained from (38). Moreover, using the following relations, (42) and (43) can be written in terms of zeta-functions:

$$
B_{2 r}=\frac{2(-1)^{r-1}(2 r) !}{(2 \pi)^{2 r}} \zeta(2 r)
$$

and $[7$, Eqs. (6.23) and (6.25)]

$$
\zeta(r, \widehat{A})=-\frac{1}{2} \frac{(2 \pi i / k)^{r}}{r !} B_{r}(0, A), r \geq 1
$$

when $r$ and $f$ have the same parity.

We also note that from Theorem 9 , the values of $\zeta(N+1, A)$ can be deduced when $N$ and $f$ have the opposite parity. For $r_{1}=r_{2}=0$ and $s=-N$ in (32), we have

$$
\begin{gathered}
H(V z,-N ; A, I ; 0,0)=A(V z,-N ; A, I)+e(-N / 2) A\left(V z,-N ; A_{-1}, I\right)=0, \\
H\left(z, s ; I, \widehat{A}_{-c} ; 0,0\right)=A\left(z,-N ; I, \widehat{A}_{c}\right)+e(-N / 2) A\left(z,-N ; I, \widehat{A}_{-c}\right)=0 .
\end{gathered}
$$

Thus, using (36), (8) and (44), equation (41) turns into

$$
\zeta\left(N+1, \widehat{A}_{-1}\right)-f(0) z^{N} \zeta(N+1)=\frac{(2 \pi i)^{N+1}}{(-k)^{N}} \sum_{m=0}^{N+2} P_{N+2-m}(0, A) P_{m}(0) z^{m-1}
$$

when $N$ and $f$ have the opposite parity.

For some special cases of $r_{1}$ and $r_{2}$ in Theorem 9, we may achieve the following formulas.

Theorem 11 Let $N$ denote a non-negative integer and $0<R<1$. Then,

$$
\begin{aligned}
& (-i \gamma k)^{-N} H(i k \gamma,-N ; A, I ;-R, 0)-k H\left(\frac{i}{k \gamma},-N ; I, \widehat{A}_{-1} ; 0, R\right) \\
& =\varphi\left(R, 0,1+N ; \widehat{A}_{-1}\right)-\frac{(2 \pi i)^{N+1}}{k^{N}} \sum_{m=0}^{N+2} P_{m}(0) P_{N+2-m}(R, A)(i / k \gamma)^{m-1}
\end{aligned}
$$


and

$$
\begin{aligned}
& k H(i k \gamma,-N ; I, \widehat{B} ; 0, R)+(-1)^{N} k \varphi(-R, 0, N+1 ; \widehat{B}) \\
& =(-i k \gamma)^{N} H\left(\frac{i}{k \gamma},-N ; B, I ; R, 0\right)-(2 \pi i)^{N+1} \sum_{m=0}^{N+2} P_{m}\left(R, B_{-1}\right) P_{N+2-m}(0)(i / k \gamma)^{m-N-1},
\end{aligned}
$$

where $\varphi(x, a, s ; A)$ is the periodic Lerch function defined by [7]

$$
\varphi(x, a, s ; A)=\sum_{n=0}^{\infty} f(n) e^{2 \pi i n x / k}(n+a)^{-s}, \text { for } \operatorname{Re}(s)>1 \text { and } x, a \text { real }
$$

in which the dash I indicates that if a is a non-positive integer, then the term corresponding to $n=-a$ is omitted from the sum.

Proof. Let $V z=-1 / z$ and put $z=i / k \gamma, \gamma>0$. Then, $R_{1}=r_{2}$ and $R_{2}=-r_{1}$. For the proof of (48), put $r_{2}=0$ and $R:=R_{2}$ and suppose that $0<R<1$ in (41). Hence, by aid of (36), we write

$$
\begin{aligned}
& \left(\frac{i}{k \gamma}\right)^{N}(-2 \pi i / k)^{-N} H(i k \gamma,-N ; A, I ;-R, 0) \\
& =(-2 \pi i / k)^{-N} k H\left(\frac{i}{k \gamma},-N ; I, \widehat{A}_{-1} ; 0, R\right)+\lim _{s \rightarrow-N} \Gamma(s) L_{-}\left(s ; A_{-1} ; R\right) \\
& \left.+e(N / 2) 2 \pi i k^{N} \sum_{\mu=0}^{k-1} \sum_{v=0}^{k-1} \sum_{m=0}^{N+2} f(-v)\right) B_{m}\left(\frac{\mu+1}{k}\right) B_{N+2-m}\left(\frac{v+R}{k}\right) \frac{(-i / k \gamma)^{m-1}}{m !(N+2-m) !}
\end{aligned}
$$

Using (6) and (7), we have

$$
\begin{aligned}
& (-2 \pi \gamma)^{-N} H(i k \gamma,-N ; A, I ;-R, 0) \\
& =(-2 \pi i / k)^{-N} k H\left(\frac{i}{k \gamma},-N ; I, \widehat{A}_{-1} ; 0, R\right)+\lim _{s \rightarrow-N} \Gamma(s) L_{-}\left(s ; A_{-1} ; R\right) \\
& +e(N / 2) 2 \pi i \sum_{m=0}^{N+2}(-1)^{m} P_{m}(0) P_{2 N+2-m}(R, A)(-i / k \gamma)^{m-1} .
\end{aligned}
$$

We must evaluate the limit above. For $0<R<1$, we have

$$
\begin{aligned}
L_{-}\left(s ; A_{-1} ; R\right) & =L\left(s ; A_{-1} ; R\right)+e^{-\pi i s} L(s ; A ;-R) \\
& =e^{-\pi i s / 2}\left\{e^{\pi i s / 2} \sum_{n=0}^{\infty} \frac{f(-n)}{(n+R)^{s}}+e^{-\pi i s / 2} \sum_{n=1}^{\infty} \frac{f(n)}{(n-R)^{s}}\right\} \\
& =e^{-\pi i s / 2}\left\{e^{\pi i s / 2} \sum_{n=0}^{\infty} \frac{f(-n)}{(n+R)^{s}}+e^{-\pi i s / 2} \sum_{n=0}^{\infty} \frac{f(n+1)}{(n+1-R)^{s}}\right\} .
\end{aligned}
$$

Now we make use of the functional equation of the periodic Lerch function [7, Theorem 6.1]

$$
\begin{aligned}
& \varphi(x, a, 1-s ; \widehat{C}) \\
& =(k / 2 \pi)^{s} \Gamma(s)\left\{e^{\pi i s / 2-2 \pi i a x / k} \varphi(-a, x, s ; C)+e^{-\pi i s / 2+2 \pi i a(1-x) / k} \varphi\left(a, 1-x, s ; C^{*}\right)\right\},
\end{aligned}
$$

where $C^{*}=\{g(-n-1)\}$ for $C=\{g(n)\}$. So, taking $a=0, x=R, C=A_{-1}=\{f(-n)\}$ and $C^{*}=\{f(n+1)\}$ in $(52),(51)$ can be written as

$$
\Gamma(s) L_{-}\left(s ; A_{-1} ; R\right)=e^{-\pi i s / 2} \Gamma(s)\left\{e^{\pi i s / 2} \varphi\left(0, R, s ; A_{-1}\right)+e^{-\pi i s / 2} \varphi\left(0,1-R, s ; C^{*}\right)\right\}
$$

which implies

$$
\lim _{s \rightarrow-N} \Gamma(s) L_{-}\left(s ; A_{-1} ; R\right)=(2 \pi / k)^{-N} e^{\pi i N / 2} \varphi\left(R, 0,1+N ; \widehat{A}_{-1}\right) .
$$


Substituting this in (50), the desired result follows.

For the proof of (49), we put $R_{2}=r_{1}=0$ and $0<R:=R_{1}=r_{2}<1$ in (38). Firstly, we evaluate the limit $\lim _{s \rightarrow-N} \Gamma(s) L_{+}(s ; B ; R)$.

Using (33) and replacing $n \rightarrow n k+v$, we have

$$
\begin{aligned}
\Gamma(s) L_{+}(s ; B ; R) & =\Gamma(s)\left\{L(s ; B ; R)+e^{\pi i s} L\left(s ; B_{-1} ;-R\right)\right\} \\
& =\frac{1}{k^{s}} \sum_{v=0}^{k-1} f^{*}(v) \Gamma(s)\left\{\zeta\left(s, \frac{v+R}{k}\right)+e^{\pi i s} \zeta\left(s, 1-\frac{v+R}{k}\right)\right\} .
\end{aligned}
$$

Now, by Hurwitz's formula for $\zeta(s, x)$ for $\operatorname{Re} s<0$ [30, p. 269] (or see [10, Eq. 3.5] for detail) the right hand-side becomes

$$
\begin{aligned}
& \frac{(2 \pi i)^{s}}{k^{s}} \sum_{v=0}^{k-1} f^{*}(v) \varphi\left(-\frac{v+R}{k}, 1-s\right) \\
& =\frac{(2 \pi i)^{s}}{k^{s}} \sum_{n=1}^{\infty} \frac{e^{-2 \pi i n R / k}}{n^{1-s}} \sum_{v=0}^{k-1} f^{*}(v) e^{-2 \pi i n v / k} \\
& =\frac{(2 \pi i)^{s}}{k^{s-1}} \sum_{n=1}^{\infty} \widehat{f^{*}}(n) \frac{e^{-2 \pi i n R / k}}{n^{1-s}}=\frac{(2 \pi i)^{s}}{k^{s-1}} \varphi(-R, 0,1-s ; \widehat{B}) .
\end{aligned}
$$

Namely,

$$
\lim _{s \rightarrow-N} \Gamma(s) L_{+}(s ; B ; R)=\frac{(2 \pi i)^{-N}}{k^{-N-1}} \varphi(-R, 0, N+1 ; \widehat{B}) .
$$

Using (37), we write

$$
\begin{aligned}
& \sum_{\mu=0}^{k-1} f^{*}\left(b\left(\mu+1+\left[R_{1}\right]\right)\right) I^{*}(z, s, 1,0,0, R) \\
& =2 \pi i k^{N} \sum_{m=0}^{N+2} B_{N+2-m}(0) k^{m-1} \frac{(-z)^{m-1}}{m !(N+2-m) !} \sum_{\mu=0}^{k-1} f^{*}(-(\mu+1)) B_{m}\left(\frac{\mu+1-R}{k}\right) \\
& =2 \pi i k^{N} \sum_{m=0}^{N+2}(-1)^{m} P_{m}\left(R, B_{-1}\right) P_{N+2-m}(0)(-z)^{m-1}
\end{aligned}
$$

where we have used that $B_{m}(1-x)=(-1)^{m} B_{m}(x)$ and (8). Substituting (53) and (54) in (38) and simplifying give (49).

Note that if we take $A=I$, the statement given by (49) in Theorem 11 reduces to [10, Theorem 3.1]. Also Theorem 3.3 of [10] can be obtained from (48) by observing that

$$
\begin{aligned}
H(z,-N ; I, I ;-R, 0) & =A(z,-N ; I, I ;-R, 0)+e(-N / 2) A(z,-N ; I, I ; R, 0) \\
& =\sum_{n=1}^{\infty} \frac{n^{-N-1}}{e^{-2 \pi i n z}-1}\left(e^{-2 \pi i n z R}+(-1)^{N} e^{2 \pi i n z R}\right)+\sum_{n=1}^{\infty} \frac{e^{2 \pi i n z R}}{n^{N+1}}, \\
H(z,-N ; I, I ; 0, R) & =\sum_{n=1}^{\infty} \frac{n^{-N-1}}{e^{-2 \pi i n z}-1}\left(e^{2 \pi i n R}+(-1)^{N} e^{-2 \pi i n R}\right) .
\end{aligned}
$$

Now, we assume that $f$ is odd or even (and of course $\widehat{f}$ ). For convenience, put $f(-m)=\delta f(m)$, where $\delta=1$ if $f$ is even and $\delta=-1$ if $f$ is odd. From (32), with (10), we have

$H(z,-N ; A, I ;-R, 0)$

$$
\begin{aligned}
& =A(z,-N ; A, I ;-R, 0)+(-1)^{N} A\left(z,-N ; A_{-1}, I ; R, 0\right) \\
& =\sum_{n=1}^{\infty} n^{-N-1} \sum_{m=1}^{\infty} f(m) e^{2 \pi i n m z / k}\left(e^{-2 \pi i n R z / k}+(-1)^{N} \delta e^{2 \pi i n R z / k}\right)+f(0)(-1)^{N} \sum_{n=1}^{\infty} n^{-N-1} e^{2 \pi i n R z / k} \\
& =\sum_{n=1}^{\infty} \sum_{v=0}^{k-1} \frac{\widehat{f}(v) n^{-N-1}}{e^{-2 \pi i(v+n z) / k}-1}\left(e^{-2 \pi i n R z / k}+(-1)^{N} \delta e^{2 \pi i n R z / k}\right)+f(0)(-1)^{N} \sum_{n=1}^{\infty} \frac{e^{2 \pi i n R z / k}}{n^{N+1}}
\end{aligned}
$$


and

$$
\begin{aligned}
H\left(z,-N ; I, \widehat{A}_{-1} ; 0, R\right) & =\sum_{n=1}^{\infty} \widehat{f}(n) n^{-N-1} \sum_{m=1}^{\infty} e^{2 \pi i n m z / k}\left(e^{2 \pi i n R / k}+(-1)^{N} \delta e^{-2 \pi i n R / k}\right) \\
& =\sum_{n=1}^{\infty} \widehat{f}(n) \frac{n^{-N-1}}{e^{-2 \pi i n z / k}-1}\left(e^{2 \pi i n R / k}+(-1)^{N} \delta e^{-2 \pi i n R / k}\right) .
\end{aligned}
$$

Thus, substituting these in (48) we have

$$
\begin{aligned}
& 2(-i \gamma k)^{-N} \sum_{n=1}^{\infty} \sum_{v=0}^{k-1} \widehat{f}(v) \frac{\cosh (2 \pi n R \gamma)}{e^{-2 \pi i(v+n i k \gamma) / k}-1} n^{-N-1}+f(0)(i \gamma k)^{-N} \sum_{n=1}^{\infty} \frac{e^{-2 \pi n R \gamma}}{n^{N+1}} \\
& =\delta \sum_{n=1}^{\infty} \widehat{f}(n) \frac{e^{2 \pi i n R / k}}{n^{N+1}}-2 k \sum_{n=1}^{\infty} \widehat{f}(n) \frac{\cos (2 \pi n R / k)}{e^{2 \pi n / k^{2} \gamma}-1} n^{-N-1} \\
& \quad-\frac{(2 \pi i)^{N+1}}{k^{N}} \sum_{m=0}^{N+2} P_{m}(0) P_{N+2-m}(R, A)(i / k \gamma)^{m-1}
\end{aligned}
$$

if $(-1)^{N} \delta=1$ and

$$
\begin{aligned}
2(-i \gamma k)^{-N} \sum_{n=1}^{\infty} \sum_{v=0}^{k-1} \widehat{f}(v) \frac{\sinh (2 \pi n R \gamma)}{e^{-2 \pi i(v+n z) / k}-1} n^{-N-1}+f(0)(i \gamma k)^{-N} \sum_{n=1}^{\infty} \frac{e^{-2 \pi n R \gamma}}{n^{N+1}} \\
=\delta \sum_{n=1}^{\infty} \widehat{f}(n) \frac{e^{2 \pi i n R / k}}{n^{N+1}}-2 i k \sum_{n=1}^{\infty} \widehat{f}(n) \frac{\sin (2 \pi n R / k)}{e^{2 \pi n / k^{2} \gamma}-1} n^{-N-1} \\
\quad-\frac{(2 \pi i)^{N+1}}{k^{N}} \sum_{m=0}^{N+2} P_{m}(0) P_{N+2-m}(R, A)(i / k \gamma)^{m-1}
\end{aligned}
$$

if $(-1)^{N} \delta=-1$.

Formulas (55) and (56) can be specialized as in Theorem 10 and are periodic analogues of [10, Theorem 3.3]. Letting $r$ tend to 0 in (55) and (56), we get Bradley's formulas (42) and (43), respectively.

We conclude the study with the following remark that mention from the relationship between periodic zeta function and Dedekind sums.

Remark 12 By comparing the definitions of $P_{r}\left(0, A_{d}\right)$ and Dedekind sum, these are closely related for $A=\{f(n)\}=\left\{P_{q}(n / k)\right\}$. More precisely,

$$
P_{r}\left(0, A_{d}\right)=k^{r-1} \sum_{m=0}^{k-1} P_{q}\left(-\frac{d m}{k}\right) P_{r}\left(\frac{m}{k}\right)=k^{r-1} s_{q, r}(-d, k),
$$

where $s_{q, r}(d, k)$ denotes the higher order (inhomogeneous) Dedekind sum.

Combining the last identity and (47) entails for $r \geq 1$ and $q \geq 2$ that

$$
\zeta\left(r, \widehat{A}_{d}\right)=(-1)^{q+1} \frac{(2 \pi i)^{r}}{2 k} s_{q, r}(d, k),
$$

when $r$ and $q$ have the same parity.

Because of this relationship, we evaluate $\widehat{A}=\{\widehat{f}(n)\}$ when $A=\{f(n)\}=\left\{P_{q}(n / k)\right\}$. From (9), we write

$$
\begin{aligned}
\widehat{f}(n) & =\frac{1}{k} \sum_{v=0}^{k-1} P_{q}\left(\frac{v}{k}\right) e^{-2 \pi i n v / k}=\frac{1}{k^{q}} k^{q-1} \sum_{v=0}^{k-1} e^{-2 \pi i n v / k} P_{q}\left(\frac{v}{k}\right) \\
& =\frac{1}{k^{q}} P_{q}\left(0, C_{n}\right)
\end{aligned}
$$


where $C=\{g(j)\}=\left\{e^{2 \pi i j / k}\right\}$ and $C_{n}=\{g(n j)\}$. From [7, Corollary 6.4], we have

$$
\zeta\left(1-q, C_{n}\right)=(-1)^{q-1}(q-1) ! P_{q}\left(0, C_{n}\right), q \geq 2 .
$$

Since

$$
\begin{gathered}
\zeta\left(s, C_{n}\right)=\sum_{m=1}^{\infty} \frac{e^{2 \pi i m \frac{n}{k}}}{m^{s}}=\varphi\left(\frac{n}{k}, s\right), \\
\varphi(x, 1-q)=-\frac{\beta_{q}\left(e^{2 \pi i x}\right)}{q}, q \geq 1 \quad \text { [[2]) }
\end{gathered}
$$

where $\varphi(x, s)=\varphi(x, 0, s ; I)$ is the Lerch function and $\beta_{q}(\alpha)$ is the Apostol-Bernoulli numbers, we have

$$
\begin{aligned}
\widehat{f}(n) & =\frac{1}{k^{q}} P_{q}\left(0, C_{n}\right) \\
& =\frac{(-1)^{q-1}}{(q-1) ! k^{q}} \zeta\left(1-q, C_{n}\right), q \geq 2 \\
& =\frac{(-1)^{q-1}}{(q-1) ! k^{q}} \phi\left(\frac{n}{k}, 1-q\right), q \geq 2 \\
& =\frac{(-1)^{q}}{q ! k^{q}} \beta_{q}\left(e^{2 \pi i \frac{n}{k}}\right), q \geq 2 .
\end{aligned}
$$

Hence, for coprime integers $d$ and $k$, this and (57) give

$$
\zeta\left(r, \widehat{A}_{d}\right)=\frac{(-1)^{q}}{q ! k^{q}} \sum_{m=1}^{\infty} \frac{\beta_{q}\left(e^{2 \pi i \frac{m}{k} d}\right)}{m^{r}}=(-1)^{q+1} \frac{(2 \pi i)^{r}}{2 k} s_{q, r}(d, k)
$$

when $r \geq 1$ and $q \geq 2$ have the same parity.

\section{References}

[1] T. M. Apostol, Generalized Dedekind sums and transformation formulae of certain Lambert series, Duke Math. J., 17 (1950) 147-157.

[2] T. M. Apostol, On the Lerch zeta function, Pacific J. Math., 1 (1951) 161-167.

[3] T. M. Apostol, Introduction to Analytic Number Theory, Undergraduate Texts in Mathematics, Springer-Verlag, New York, 1976.

[4] B. C. Berndt, Generalized Dedekind eta-functions and generalized Dedekind sums, Trans. Amer. Math. Soc., 178 (1973) 495-508.

[5] B. C. Berndt, Character transformation formulae similar to those for the Dedekind etafunction, Proc. Sym. Pure Math. XXIV, Amer. Math. Soc., Providence, (1973) 9-30.

[6] B. C. Berndt, Generalized Eisenstein series and modified Dedekind sums, J. Reine Angew. Math., 272 (1975) 182-193.

[7] B. C. Berndt, Periodic analogues of the Euler-Maclaurin and Poisson summation formulas with applications to number theory, Acta Arith., XXVIII (1975) 23-68.

[8] B. C. Berndt, On Eisenstein series with characters and the values of Dirichlet L-functions, Acta Arith., XXVIII (1975) 299-320.

[9] B. C. Berndt, Reciprocity theorems for Dedekind sums and generalizations, Adv. in Math., 23 (1977) 285-316. 
[10] B. C. Berndt, Modular transformations and generalizations of several formulae of Ramanujan, Rocky Mountain J. Math., 7 (1977) 147-190.

[11] B. C. Berndt, Analytic Eisenstein series, theta functions and series relations in the spirit of Ramanujan, J. Reine Angew. Math., 303/304 (1978) 332-365.

[12] D. M. Bradley, Series acceleration formulas for Dirichlet series with periodic coefficients, $R a$ manujan J., 6 (2002) 331-346.

[13] M. Can and V. Kurt, Character analogues of certain Hardy-Berndt sums, Int. J. Number Theory, 10 (2014) 737-762.

[14] L. Carlitz, Generalized Dedekind Sums, Math. Z., 85 (1964) 83-90.

[15] M. Cenkci, M. Can and V. Kurt, Degenerate and character Dedekind sums, J. Number Theory, 124 (2007) 346-363.

[16] M. C. Dağlı and M. Can, On reciprocity formula of character Dedekind sums and the integral of products of Bernoulli polynomials, J. Number Theory, 156 (2015) 105-124.

[17] M. C. Dağlı and M. Can, Periodic analogues of Dedekind sums and transformation formulas of Eisenstein series, http://arxiv.org/abs/1506.01809.

[18] L. A. Goldberg, Transformations of theta-functions and analogues of Dedekind sums. Ph.D. thesis, University of Illinois, Urbana (1981).

[19] Y. Hamahata, Dedekind sums with a parameter in finite fields, Finite Fields Appl., 28 (2014) 57-66.

[20] K. Katayama, Ramanujan's formulas for L-functions, J. Math. Soc. Japan, 26 (1974) 234240.

[21] K. Katayama, Zeta functions, Lambert series and arithmetic functions analogous to Ramanujan's $\tau$-function, J. Reine Angew. Math., 268/269 (1974) 251-270.

[22] J. Lewittes, Analytic continuation of the Eisenstein series, Trans. Amer. Math. Soc., 171 (1972) 469-490.

[23] S. G. Lim, Generalized Eisenstein series and several modular transformation formulae, $R a-$ manujan J., 19 (2009) 121-136.

[24] J. L. Meyer, Character analogues of Dedekind sums and transformations of analytic Eisenstein series, Pacific J. Math., 194 (1) (2000) 137-164.

[25] Y. Nagasaka, K. Ota and C. Sekine, Generalizations of Dedekind sums and their reciprocity laws, Acta Arith., 106 (2003) 355-378.

[26] H. Rademacher and E. Grosswald, Dedekind sums, Carus Math. Monographs, Vol. 16, Math. Assoc. Amer., 1972.

[27] C. Sekine, Dedekind sums with roots of unity and their reciprocity laws, Tokyo J. Math., 26 No:2 (2003) 485-494.

[28] C. Sekine, On Eisenstein series with characters and Dedekind sums, Acta Arith., 116 (2005) $1-11$.

[29] L. Takacs, On generalized Dedekind sums, J. Number Theory, 11 (1979) 264-272.

[30] E. T. Whittaker and G. N. Watson, A course of modern analysis, 4th ed., University Press, Cambridge, 1962. 